\title{
Erratum to: Bayes optimal template matching for spike sorting - combining fisher discriminant analysis with optimal filtering
}

\author{
Felix Franke $^{1,2} \cdot$ Rodrigo Quian Quiroga ${ }^{3} \cdot$ Andreas Hierlemann $^{1} \cdot$ Klaus Obermayer $^{2}$
}

Published online: 15 April 2015

(C) Springer Science+Business Media New York 2015

Erratum to: J Comput Neurosci

DOI 10.1007/s10827-015-0547-7

In the original version of the article, the following equations in the appendix were incorrectly displayed.

\section{Matched filtering}

First inline equation: $y(t)=\sum_{i} f_{i} * x(t)$

Derivation of Bayes Optimal Template Matching Incorrect line break in inline equation: $f_{i}=\boldsymbol{C}^{-1} \xi_{i}$

Definition of $c_{i}: c_{i}=-\frac{1}{2} \xi_{i}^{T} \boldsymbol{C}^{-1} \xi_{i}+\ln (p(i))$

\section{Discriminant function for noise}

Equation (8): $d_{n}(t)=X(t)^{T} \boldsymbol{C}^{-1} 0-\frac{1}{2} 0^{T} \boldsymbol{C}^{-1} 0+\ln (p(n))$

Equation (9): $d_{n}(t)=t h r=d_{n}=\ln \left(1-\sum_{i} p(i)\right)$

1 ETH Zürich, Department of Biosystems Science and Engineering, Basel 4058, Switzerland

2 School for Electrical Engineering and Computer Science, Technische Universität Berlin, Berlin, Germany

3 Centre for Systems Neuroscience, University of Leicester,

Leicester, UK 